Resumo de Tese

\title{
A associação entre o perfil clínico e psicossocial de pessoas com diabetes mellitus usuárias de uma unidade de saúde da família de Sorocaba - SP \\ These Abstract
}

\section{The association of the clinical and psychosocial profile of diabetes mellitus patients using a Family Health Unit in Sorocaba - SP}

Adriana Cecel Guedes

\section{Resumo}

Diabetes Mellitus (DM) é uma doença crônica responsável por altos índices de morbi-mortalidade. Diversas tentativas de intervenção vêm sendo feitas o controle dessa doença e para diminuir a sua incidência das doenças crônicas, entretanto os números ainda são bastante assustadores. A atenção primária à saúde é o âmbito privilegiado para o acompanhamento das pessoas com diabetes mellitus (DM) especialmente no Programa de Saúde da Família que constitui estratégia prioritária para a reestruturação do modelo assistencial vigente no Sistema Único de Saúde. Assim, compreender os aspectos psicossociais e das condições de saúde das pessoas com DM pode ser importante nesse sentido. Esse trabalho teve por objetivos: Identificar como as pessoas com diabetes mellitus usuárias de uma Unidade da Saúde da Família (USF) no interior de Saõ Paulo avaliam seu cotidiano, seu lazer, seu trabalho e os seus relacionamentos; caracterizar as condições clínicas e o estilo de vida dessas pessoas e verificar a relação entre a auto-avaliação do cotidiano, lazer, trabalho e relacionamento, suas condições clínicas, e os seus estilos de vida. Trata-se de um estudo não experimental, correlacional com uma abordagem quantitativa. Foram avaliadas 152 pessoas com diabetes usuárias de uma USF em Sorocaba-SP por meio de respostas a questões de um questionário, pelo exame físico e pela avaliação dos prontuários. A amostra foi composta, em sua maioria, por pessoas do sexo feminino $(59,2 \%)$, com idade média de 58,22 anos, casadas $(57,9 \%)$, e católicas $(58,6 \%)$. Possuíam baixo nível de escolaridade e baixa renda mensal sendo que $48 \%$ não exerciam atividade formal de trabalho. O tempo médio do diagnóstico de DM foi de 9,25 anos. A maior parte da população, (42,7\%) utilizava hipoglicemiantes orais como terapêutica medicamentosa. A presença de complicações crônicas foi referida por $44,2 \%$ dos participantes da pesquisa, sendo a retinopatia a mais freqüente entre elas. $86,7 \%$ apresentavam controle glicêmico ruim e a maioria estava com sobrepeso. As pessoas fizeram uma regular avaliação dos aspectos psicossociais atribuindo melhor média aos relacionamentos sociais e a pior média ao lazer. Quanto ao estilo de vida e ao autocuidado mais da metade das pessoas referiram acordar disposto após uma noite de sono, 77,6\% referiram não fumar ou ingerir bebidas alcoólicas, $50 \%$ dos participantes da pesquisa não praticavam atividades físicas, e a maior parte das pessoas estudadas fazia automonitorização de forma irregular na farmácia ou na UBS. Homens e mulheres que tinham maior nível de escolaridade avaliaram melhor os aspectos psicossociais de suas vidas e praticavam de forma correta a auto monitorização, pessoas mais velhas fumavam mais e ingeriam mais bebidas alcoólicas. As pessoas com menor renda mensal apresentavam maiores dificuldades para dormir enquanto as pessoas que melhor avaliaram suas vidas dormiam melhor e praticavam mais atividade física da forma adequada. As pessoas que praticavam atividades

Palavras-chave: Diabetes mellitus; Estilo de vida; Impacto psicossocial.
Key Words: Diabetes mellitus; life style;

psychosocial impact 
físicas tinham melhor controle glicêmico. Assim, foi possível identificar que as condições psicossociais e sociodemográficas podem estar ligadas às condições de saúde das pessoas com DM, o que deve ser considerado pelos profissionais que atuam na USF, visando à promoção e a proteção da saúde, prevenção de agravos, o tratamento e a manutenção da saúde das pessoas com diabetes. Desse modo recomenda-se o incremento de estudos que mostrem a influência dos aspectos psicossociais no controle do DM e de outras doenças crônico degenerativas, tornando esse tema base para a elaboração de estratégias de intervenção diferenciadas para a promoção da saúde das pessoas.

Dissertação de Mestrado 2007 Universidade de São Paulo - Enfermagem Na Saúde Do Adulto

\section{Disponivel para consulta em:}

http: / / servicos.capes.gov.br/capesdw / resumo.html?idtese $=2007633002010186 \mathrm{P} 6$ 